\title{
Commotio Cordis and Contusio Cordis: Possible Causes of Trauma-Related Cardiac Death
}

\author{
Ali Reza Farrokhian ${ }^{1, *}$ \\ ${ }^{1}$ Trauma Research Center, Kashan University of Medical Sciences, Kashan, IR Iran \\ "Corresponding author: Ali Reza Farrokhian, Trauma Research Center, Kashan University of Medical Sciences, Kashan, IR Iran. E-mail: farrokhian.ar@gmail.com
}

Received 2016 August 13; Revised 2016 September 24; Accepted 2016 September 25.

Keywords: Commotio Cordis, Contusio Cordis, Death

An important complication of chest trauma is serious cardiac problems. The fatal conditions like cardiac arrhythmias, including ventricular fibrillation and sudden cardiac arrest can occur in both cardiac concussion (commotio cordis) and cardiac contusion (contusio cordis). Commotio cordis is to be distinguished from cardiac contusion (contusio cordis) or blunt cardiac injury, a condition in which blunt chest trauma causes structural cardiac injury, such as those observed in road traffic accidents (1). In this brief, it will be tried to draw attention of trauma specialists, nurses, paramedics and other persons involved in trauma care to these two serious complications of chest trauma that sometimes are misunderstood.

Commotio cordis is a Latin meaning "agitation of the heart". It is defined as sudden cardiac death secondary to relatively low-impact blunt trauma to the anterior chest wall. Sudden cardiac death following chest trauma has been reported in the medical literature since the middle of the 18th century in the context of chest trauma among workers $(1,2)$. While the accurate incidence of these nonstructural cardiac injuries is unknown, mostly due to under-reporting of cases, commotio cordis has been reported as the second most common cause of sudden death in athletes, especially in baseball and hockey players (sports with blunt projectiles) and football players (a sport with more physical body contact) (2). Young males are mainly affected. Mean age of victims is 15 and only 9 percent of the reported cases were older than 25 years ( 1 , 2). Despite its traumatic manifestation, sudden death due to "commotio cordis" appears to be a primary electrical incident, with ventricular fibrillation (VF) occurring immediately upon chest wall impact (3). The most important variable in the development of VF in the cases of commotio cordis appears to be the timing of blunt chest trauma within the cardiac cycle. Only impacts occurring during a 20 to 40 millisecond window on the upslope of the T-wave (early ventricular repolarization) will cause VF (4). Other factors, like location of impact (directly over the cardiac silhouette), velocity of impact [as projectile velocity increases up to 40 miles per hour (mph), the incidence of VF increases to 70\%] (5), hardness of an impact object (harder objects are more likely to cause $\mathrm{VF}(6)$ and shape of an impact object (flat objects have never caused VF in the experimental model, and smaller diameter spheres are more likely to cause VF) (7) are also important factors to cause the problem.

Unlike "commotio cordis" where the structural damage of the heart does not happen, in "contusio cordis", contusion of myocardial muscle, rupture of a cardiac chamber, or disruption of a heart valve may be caused by blunt trauma to the chest mostly due to motor vehicle accidents. Myocardial contusion may be minor and asymptomatic, although tachycardia may be present. Some patients develop conduction abnormalities and/or dysrhythmias. Ventricular rupture is usually rapidly fatal, but patients with smaller, particularly right-sided, lesions may survive to present with cardiac tamponade. Tamponade due to atrial rupture may manifest more insidiously. Valve disruption may occur, causing a heart murmur and sometimes manifestations of heart failure (e.g. dyspnea, pulmonary crackles, sometimes hypotension), which may develop rapidly. Septal rupture may not cause symptoms initially, but patients may present later with heart failure.

Following the identification of sudden cardiac arrest, the management of persons with commotio cordis follows the standard basic and advanced life-support algorithms. Management should include chest compressions with early defibrillation, as indicated.

In contusio cordis, prehospital management depends upon patient symptoms and severity of illness. Prehospital providers should treat patients with possible severe cardiac injury, according to the principles of Advanced 
Trauma Life Support®, paying special attention to the patient's airway, breathing, and circulation. Diagnosis of blunt cardiac injury (BCI) in the field can be difficult. Trauma patients may have multiple injuries and signs suggestive of BCI, such as arrhythmia, which may stem from hypotension, acidosis, or preexisting heart disease. For patients with minor injuries and no respiratory difficulty, chest pain, or other concerning symptoms, no intervention may be needed.

In conclusion, it must be reiterated that in case of encountering accidental injuries any abnormality in heart function should be attributed to either " commotion cordis " or " contusion cordis " and thus it can be hopeful to prevent probable death produced by these causes.

\section{References}

1. Link MS. Commotio Cordis Ventricular Fibrillation Triggered by Chest Impact-Induced Abnormalities in Repolarization. Circulation: Arrhythmia Electrophysiol. 2012;5(2):425-32. doi: 10.1161/circep.111.962712.
2. Maron BJ, Estes N3. Commotio cordis. NEngl J Med. 2010;362(10):917-27. doi:10.1056/NEJMra0910111. [PubMed: 20220186].

3. Link MS, Estes NM, Maron BJ. Eligibility and Disqualification Recommendations for Competitive Athletes With Cardiovascular Abnormalities: Task Force 13: Commotio Cordis A Scientific Statement From the American Heart Association and American College of Cardiology. Circulation. 2015;132(22):339-42.

4. Madias C, Maron BJ, Weinstock J, Estes N3, Link MS. Commotio cordissudden cardiac death with chest wall impact. J Cardiovasc Electrophysiol. 2007;18(1):115-22. doi: 10.1111/j.1540-8167.2006.00684.x. [PubMed: 17229310].

5. Link MS, Maron BJ, Wang PJ, Vander Brink BA, Zhu W, Estes NA. Upper and lower limits of vulnerability to sudden arrhythmic death with chest-wall impact (commotio cordis). J Am Coll Cardiol. 2003;41(1):99104. doi: 10.1016/S0735-1097(02)02669-4.

6. Link MS, Wang PJ, Pandian NG, Bharati S, Udelson JE, Lee MY, et al. An experimental model of sudden death due to low-energy chestwall impact (commotio cordis). N Engl J Med. 1998;338(25):1805-11. doi: 10.1056/NEJM199806183382504. [PubMed: 9632447].

7. Kalin J, Madias C, Alsheikh-Ali AA, Link MS. Reduced diameter spheres increases the risk of chest blow-induced ventricular fibrillation (commotio cordis). Heart Rhythm. 2011;8(10):1578-81. doi: 10.1016/j.hrthm.2011.05.009. [PubMed: 21699851]. 